\title{
Leadership in context: Insights from a study of nursing in Western
}

\author{
Australia \\ Linley Lord, Therese Jefferson, Des Klass, Margaret Nowak, and Gail Thomas \\ Curtin University, Perth, Australia
}

\begin{abstract}
This paper investigates the importance of integrating context when analysing the role and practice of leadership within a specific organization or profession. It does this with reference to a study of nursing in Western Australia. Using theoretical sampling, qualitative data were collected through interviews and focus groups with targeted stakeholders in Western Australia's public health system. The main purpose of the data collection and analysis was to identify perceptions and understandings of leadership among key stakeholders. Findings emerged which identified the importance of considering specific dimensions of the cultural, social and institutional context in order to understand the practice and experience of leadership among nurses in the Western Australian public health sector.
\end{abstract}

\section{Keywords}

Leadership, nursing leadership, leadership in context 


\section{Introduction}

The objective of the research was to contribute a detailed understanding of contemporary nursing and midwifery leadership in the WA public health system. This was achieved by considering the perceptions of nurses and midwives about the practice and the issues for nursing leadership. The key information and data sources for the research consisted of existing literature and reports, and interview and focus group discussions with key stakeholders.

Leadership as a subject has been extensively researched. Despite this, it remains a term that defies easy definition and those definitions which do exist are highly contested (Grint, 2005). One of the key, relatively recent insights to emerge from this literature, however, is the importance of organizational context in the study of leadership (see for example, Ladkin, 2010). During data analysis in our study, context emerged as an important dimension for developing an understanding of perceptions of and practices in nursing leadership within the Western Australian public health sector. This paper focuses on the contribution of contextual dimensions to our understanding of the practice of leadership among nursing professionals in the Western Australian public health sector. 
Our investigation of leadership and context draws specifically on Jepson's (2009) identification of contextual dimensions of leadership and qualitative analysis of interview data with nurses and other stakeholders in Western Australia's public health sector. This approach facilitates the identification of important contextual factors that emerged in our study of nursing and leadership. The analysis suggests that a more complete understanding of the issues that constrain and facilitate leadership can be gained by considering literature that, so far, has not been viewed as central to the study of leadership among nursing professionals. The study's findings support the value and importance of integrating contextual factors when attempting to understand the role of leadership within a specific organization or profession.

\section{Contextual dimensions of leadership}

Historically, researchers have seen leadership as a function of individual agency, and have focused on characteristics and abilities (trait approaches), actions (behavioural approaches) or particular roles and structures (Bolden, 2011). In relative terms, research has tended to disregard context as an important element of understanding leadership roles and practices (Sarros, 2009). However, leadership does not happen in a vacuum and must be understood in a social context. It is socially constructed and is 
embedded in a context where patterns of beliefs and values currently and over time must be considered and where history also matters (Osborn et al., 2002).

Several dimensions of context have been identified in the literature as having an influence on leadership. To date, culture and climate have received the lion's share of attention from researchers as the most significant contextual influences on leadership (Jackson and Parry, 2008). More recently, the identification of potentially relevant dimensions of context has been examined in detail by Jepson (2009), who identifies three different levels of context that interact with each other. Figure 1 summarises these various contextual dimensions.

Insert Figure 1 here

An overarching framework which provides an integrated approach to exploring contextual dimensions of leadership has several advantages for studies of leadership in nursing. The dimensions developed by Jepson provide one lens through which to consider the various contextual dimensions in which leadership is practiced. In the 
study outlined below, it provides a flexible but systematic framework for considering findings relevant to the practice and development of leadership in the profession of nursing.

It is evident that nursing scholars, like many others, have struggled to define the concept of leadership. There is a plethora of definitions of leadership within literature about nursing (Stanley, 2006b; Watson, 2008). For example, leadership is the art of influencing a group of others towards the achievement of common goals (Calpin-Davies, 2003; Cummings et al., 2008; McArthur, 2006); or unifying people around values and then constructing the social world for others around those values and helping them get through change (Stanley, 2006b). It has also been defined as identifying goals and providing support and motivation to achieve them (Davidson et al., 2006); empowering individuals and teams (Pinch and Della, 2001) or articulating a vision, and working with others to transform that vision into reality (Woods, 2003).

While many definitions of nursing leadership have been taken from business and social science literature (Davidson et al., 2006; Laurent, 2000), there have also been attempts to craft a nursing-specific definition. One characteristic of these approaches is reference to the contextual characteristics of nursing, particularly the communicative and instrumental care roles played by nurses within large 
organizations. For example, Chadwick (2010: 159) defines nursing leadership as 'the ability to represent masses of people by facilitating dialogue between various levels of professional and non-professional staff'. Carney (2009: 436) believes that 'leadership means providing healthcare through a collaborative and ethical process that uses advocacy to effect change for the benefit of patients'. The Canadian Nurses Association suggests that 'nursing leadership is about the competent and engaged practice of nurses, who provide exemplary care, think critically and independently, inform their practice with evidence, delegate and take charge appropriately, advocate for patients and communities, insist on practising to their full and legal scope and push the boundaries of practice to innovative new levels' (Canadian Nurses Association, 2009).

The 'nursing specific' definitions suggest, sometimes implicitly, that it is difficult to understand and analyse the concept of leadership within the nursing profession without investigating key features of the specific workplace context in which patients are located and nursing work occurs. As demonstrated above, definitions of leadership appear to become particularly meaningful when they integrate key contextual features of the type of role performed by nurses and the environment within which they practice. 
The purpose of this paper is to explore the importance of contextual dimensions for understanding nursing leadership. This exploration is done with reference to a qualitative study of leadership among nurses and midwives in the Western Australian public health sector.

\section{Methodology and approach}

The study was based in the qualitative paradigm; it was the phenomenon of leadership in nursing that was being explored. The sample used focussed on nurses in the WA public health sector and theoretical sampling was used in an inductive sense to develop the leadership framework.

Two levels of analysis were employed in the final study. The first phase of the study is the subject of this paper. This phase used interviews and focus groups to provide qualitative data to enable the research team to surface the relevant leadership constructs within context for further investigation. The second phase involved a survey to verify the constructs surfaced in phase one.

Qualitative data were gathered via in-depth semi structured interviews with senior nurse managers, nurses and doctors within the public health setting. In addition focus groups of eight to 10 participants were conducted using Group Support System (GSS) technology. 
The necessary interview protocols were observed and interviewers were sensitive to the importance of bracketing. Open ended questions were developed to encourage the surfacing of participant's lived experience of nursing leadership. Twenty-two interviews were conducted and 64 participated in the GSS based focus groups.

NVivo was used as the data management tool for the qualitative data to ensure we had a robust audit trail. A thematic approach was used to structure the analysis of the qualitative data and rigor protocols for authenticity and trustworthiness were adopted. To limit research bias the team utilized bracketing throughout the data collection and analysis process. Several team meetings were conducted to discuss codes and allow for constant comparisons in the analysis.

\section{The emergence of context in discussions of leadership}

Although not identified as an initial objective of this project, one of the key findings to emerge from the analyses was the focus of participants on key contextual factors when they discussed leadership among nurses and midwives. This focus was particularly relevant to the first part of the analysis which relied directly on open coding to identify the key themes that emerged from participants' perceptions and descriptions of leadership in their workplaces. The effect of these emergent research 
themes was twofold. Firstly, as outlined above, the project was considered more purposefully in terms of the developing literature on leadership and context. Secondly, the data analysis was used to inform a subsequent targeted literature search which focused on key contextual factors influencing leadership practice among nursing but which, to date, have rarely been considered as an integral part of leadership research. In keeping with the inductive nature of this study, this latter literature forms part of the discussion of results.

In our presentation of findings below, we discuss the emergent contextual themes from the open coding analysis process. Systematically considering these contextual aspects of leadership practice serves to demonstrate and define some key issues specific to nursing leadership in Western Australia's public health sector.

In presenting our findings, we consider our analysis within the overarching framework provided by Jepson. This approach gives explicit focus on contextual dimensions that is helpful for understanding key issues that inform leadership practice among nurses in our study. Specifically, a focus on social, institutional and cultural contextual dimensions assists with identifying and analysing how an ethic of care is particularly important to understanding leadership practice among nurses. An explicit consideration of Jepson's framework alongside the emergent themes also serves to 
identify key dimensions not specifically included in Jepson's discussion, such as geographic and intergenerational factors.

The discussion below provides an indicative selection of empirical material that illustrate the key contextual themes that were identified. In some cases specific words have been slightly altered to protect the identity of the participant. Text that has been changed for this purpose has been enclosed in square brackets. At all times, care has been taken not to alter the meaning of the text in any substantive manner. Clarifying text added by the researchers is included in bracketed, non-italicised text.

\section{Immediate social context}

We have clustered our first group of emergent themes under the heading of 'immediate social context'. This term is used by Jepson (2009) to describe the daily context in which work is undertaken including the job being undertaken and the industry, organization, group, hierarchy and/or department in which it is located. It also refers to technology relevant to the work environment.

\section{Role clarity and conflict}

In this study, there was extensive discussion by participants about the lack of role clarity that existed in their workplaces and the way in which this impeded effective 
decision-making and leadership. Aspects of the present organization of the delivery of nursing roles, particularly within the larger, predominantly metropolitan, hospitals were seen by our respondents as being inimical to the provision of appropriate leadership. Interview participants discussed confusion about lines of authority. The organizational context seemed, to some participants, to define very specific roles to specific tasks and thus removed the potential to exercise leadership, resulting in some paradoxical effects. On the one hand, while the existence of multiple, tightly defined roles might be expected to contribute to clear lines of decision-making authority, it appeared to make it difficult to know where authority and accountability resided for specific decisions. On the other hand, very tight role definitions appeared to make it clear who was not responsible for particular decisions rather than who could assume a decision-making role:

Extract 1: some of it is about lack of clarity about what your role is...

Extract 2: We need to look at what is the definition of clinical management and I often hear from senior nurses 'that's not my responsibility because it is not clinical'.. 
This meant that organizational lines of authority contributed to a perception that it was difficult to find people who could take an integrated, holistic approach to decisions within the organization.

Extract 3: Where people are thinking this is only my role, that's not my role;...as a leader you have got to think of all the roles in one...and yet all the roles are intertwined, educator, manager, clinical person, leader, all in one.

The comments of respondents suggest that identifiable leadership roles are not clear to nurses and, associated with this, the capacity for some individuals to exercise effective leadership was constrained. As a result respondents identified confused or tangled lines of accountability and in some instances failure of accountability, inadequate or confused communication and fragmented procedures for the delivery of care, especially in respect of the linkage between appropriate types of resourcing and the specific resource needs of care givers. Inadequacies in decision making and difficulties in leading responses to the changing requirements for care are a result. Informal leadership has developed as one way addressing these challenges. 
Extract 4: The informal leadership keeps that [ward] going on a regular basis...Philosophy and passion to keep going...

Extract 5: ...on the coalface I think probably the informal leadership is...the stronger leadership...these people who get things done, have the knowledge, seek out the knowledge...

Other issues relating to role included opportunities for 'role drift', whereby the tasks undertaken relate to the interests of individuals rather than the needs of the team and the proliferation of role titles that occurs within the health system.

Extract 6: ...over time it's evolved because of the skill the person brought to the position...rather than us saying we want you to do this role.

Extract 7: ...each site has done it individually, called it differently, ... now we've got this hotch potch of different positions, or positions with different titles... 
Issues relevant to role clarity have been identified previously in studies of nursing but this issue is not always explicitly linked to the potential implications this has for leadership. However, our findings suggest that this has important implications for leadership practice. We return to this matter in our discussion.

\section{Management and leadership}

Possibly associated with a lack of role clarity, participants described confusion about the perceived differences between leadership and management and the links between the two. The perception often given was that management roles lacked status relative to clinical roles; management was downplayed and not perceived to provide opportunities for the exercise of leadership in nursing. This perception leads to a distinctive feature of the context in which nursing leadership is practiced; nursing leadership is perceived by some participants to be quite separate from and in some cases precluded by undertaking a management role. That is, management does not necessarily involve leadership attributes or skills. When this distinction was made there was a tendency to consider direct clinical practice as an implicit requirement (necessary but not sufficient) for leadership.

Extract 8: Clinical leadership and nurse managers are different. 
Extract 9: Nurse Manager[s]... are only managing the staff component, leave, rosters, resources, so they are more the doer rather than leading...

Extract 10: ...[I] see them [management and leadership] as two separate things .... think anybody can be given the tools to actually get the job [of management] done because it is quite routine.

Extract 11: ...management is, it's more task oriented and its more about making sure you meet certain targets...is sort of measurable really...different from leadership.

Extract 12: Managers carry out day to day functions, leadership is more strategic.

The clear distinction between management and leadership provided a challenge for some nursing leaders interviewed. They also perceived that leadership was associated with clinical roles rather than managerial roles. For some leaders, this resulted in a perceived need to reinforce or re-establish their clinical credentials by reengaging with clinical roles in order to effectively practice leadership within the organization: 
Extract 13: I have an SRN 7 and he said 'I'd like to go and work on a ward' and I said, 'yes, why?' And he said 'for credibility'.

For other respondents, however, while leadership functions differ from management tasks, they perceive the two to be interrelated and see providing management leadership as an important component in developing the conditions of trust and the resourcing required to support staff and implement change.

Extract 14: You can manage an organization for a short while without leading, but it doesn't last very long.

Extract 15: I've always thought it was a false dichotomy between leader and manager... [they] overlap more than they don't overlap.

The lack of clarity about the distinction between manager and leader roles is widely recognised in previous research and, is more overtly linked with dialogues about leadership than the previous theme of role clarity (see for example Carney, 
2009; Carryer et al., 2007; McCallin and Frankson, 2010; New South Wales Health, 2009; Stanley, 2006a; Williams et al., 2009). Despite the recognition of the challenges associated with identifying and distinguishing between managerial and leadership roles, successful policy measures to address this challenge remain elusive. Findings from our project suggest that this difficulty may be related to policies affecting the practice of public sector management in general and health sector management in particular. The links between models of public sector management and nursing leadership are not well researched. However, our findings suggest that this contextual dimension has important implications for nursing leadership and we return to this theme in our discussions.

\section{Mental health and midwifery}

In Jepson's framework, 'departments' are identified as a potential contextual factor that might have implications for the specific contextual dynamics relevant to leadership practice. The importance of specific departments or areas of operational activity emerged in our study with reference to nurses working in mental health and midwifery.

One of the concerns raised was the issue of 'voice'; concern that decisions affecting mental health nurses and midwives are sometimes made without reference 
to their areas of practice. This lack of voice was coupled with a perception that those who do not have a background specific to their area of operation, whether it be mental health or midwifery, are not equipped to provide leadership and make appropriate decisions for issues specific to that area. In addition, there was a perception that these parts of the hospital system were somehow less visible or neglected in the priorities of the broader organization.

Extract 16: If you don't have a mental health or a midwifery background you're disadvantaged ...talking about issues specifically relating to those areas

Extract 17: If there is [a mental health voice] it is ad hoc.

Extract 18: We always come second...mental health is always an afterthought.

\section{Regional and remote contexts}

While geography is not a specific factor listed in Jepson's contextual dimensions of leadership, it emerged as an important theme among some of our interview and focus group participants. As mentioned in our description of the data collection process, our study included participants from two regional areas, including nurses and midwives 
from remote nursing posts, small district hospitals, and regional hospitals. Geographical location was important for some of the findings that emerged from the data analysis.

Among participants, there was an impression that leadership is more 'immediate' in country settings; there is limited opportunity to defer decision-making and leadership practices to others. This sense of immediacy was perceived as both a strength and weakness of regional and remote organizational centres. If appropriate leadership is not provided the local impact could be very direct and quite problematic.

Extract 19: More delegation happens in the country that's concentrated in a smaller number of people...you don't have strong leaders then everything in the whole house of cards falls down.

Extract 20: Nursing 'structure' can get in the way of flexibility to provide remote care needed on the ground.

In some ways, participants in regional and remote centres described situations that appeared to be a microcosm of the larger hospital system. In common with metropolitan based discussions, participants identified a need for flexibility and 
autonomy and felt that this was not encouraged or accommodated with tightly defined, system-wide management approaches to fulfilling particular roles and tasks. There was some frustration expressed about system wide factors which impede leadership.

Extract 21: For us to be good leaders we need to be able to change the things that don't work instead of being told 'this is the system, you will work with it'. Extract 22: [WA is a] very diverse state and I don't think it is really recognised enough with it still being very 'hospital' centred.

Despite the differences, clinical credibility was seen as crucial to leadership in regional and remote areas, and the comment from one Director of Nursing resonates with findings from metropolitan interviews:

Extract 23: On a small site, a multipurpose site we [Director of Nursing] have to have that clinical credibility. 
Points raised at the departmental and geographical levels such as lack of 'voice', system wide rules overriding or obstructing local needs and perceptions of disregard for operational issues at the individual workplace can be understood with the perspective of our subsequent discussion of the importance of understanding the context of public sector management in the health sector as a filter for respondents' perceptions.

\section{Institutional}

Jepson uses the term 'institutional' to refer to features such as the history, education, regulation and socialization relevant to specific leadership contexts. These types of categories were evident in two specific themes that emerged from our data analysis. The first is leadership development and succession planning, which might broadly be considered forms of or strategies for education. The second theme concerns intergenerational issues which, while also related to education, are likely to also include some socialization factors.

\section{Leadership development and succession planning}

There was considerable discussion about the need to identify potential leaders and ensure their skills development through mentoring and learning opportunities. The issue was seen as taking on extra urgency because of the age structure of nursing and 
the expectation that many existing nursing leaders are moving towards, retirement. Many stressed the important role for current leaders in both identifying and mentoring/coaching future leaders. Some also saw the need for more support for formal education and/or accredited courses.

Extract 24: Grow your own [leaders]...[we] should be growing the young nurses into managers or leaders.

Extract 25: Recognising the good leaders, potential leaders, pulling them out and empowering them

Extract 26: Succession planning historically something we have not done well...making sure the next people coming along actually do have the skills.

Extract 27: People don't have the skills...need to give them time to mentor, to be mentored and be coached.

It was noted that in some cases, especially in rural and regional areas, an absence of structured succession planning, education and training led to a 'last person standing' process of succession. For some it seemed that it was expected that 
leadership skills and the role would just happen on-the-job. Some respondents noted that, while support for the development of clinical skills and knowledge was available, development of leadership potential in nurses was unsuitable/lacking.

Extract 28: There is an expectation the people will just learn things as they come up.

Extract 29: Clinical leaders...we've expected them to have leadership attributes, leadership skills to be able to cope with the reform and they actually haven't.

A related institutional leadership issue raised by a number of rural and regional nurses, but also an issue for metropolitan nurses, was the very high prevalence of acting positions at 'leadership' levels and the excessive time taken to select and appoint. It was noted that this situation created paralysis in the ability to lead and make changes, was demotivating for those with applications for position or appointed for long periods in acting positions, and reduced staff confidence in those in leadership roles who were in an acting position only. Comments included:- 
Extract 30: [You] can't expect people to lead knowing it's only for three months and then another three months.

Extract 31: People are interviewed and they are appointed acting for three months...annoys them...they know they have to apply for it again.

In some respects, the issues raised in this theme were closely related to organizational issues and reflected leadership and system 'failures'. They are also distinguished by the identification by participants of ways in which leadership is 'learned' in their organization.

\section{Intergenerational leadership challenges}

Intergenerational issues are not specifically included in Jepson's typology. However, we have interpreted this broadly as a 'socialization' dimension that relates to differences between different cohorts of nurses. To understand some of the comments below, it is important to realise that nursing education in Australia underwent significant change between the late 1970s and 1993 when registered nurse education completed the transition from being hospital-based to being university 
based. Participants noted that there were challenges for nursing leadership around the related expectations of tertiary educated Generation $Y$ nurses. These challenges were around three aspects, not all of which can be specifically linked to a university education but which seemed to be broadly linked within some participants' discussions. The first was the perceived differences in Gen $Y$ lifestyle choices and the challenges and issues for nursing leaders in responding to/accommodating these. The second related to differences in leadership style expectations of current leaders and Gen $Y$ nurses; these were in part related to the confidence and knowledge of the latter. The third relates to the willingness of younger nurses to take on leadership roles and, related to this, some evidence of resentment of such leaders by some older nurses.

Extract 32: Gen $X, Y$, don't give them their Saturday afternoon off...they vote with their feet.

Extract 33: It's about being mindful that these people coming through are different...older nurses in clinical positions...a little bit apprehensive because they are having to change the way they manage and lead...some of our senior nurses struggle with that. 
Extract 34: Newly educated ones might be a bit challenging...very well informed about things.

Extract 35: I don't think everybody thinks the same about leadership; the expectations of the new nurses...is different to the older nurses.

\section{Cultural}

Jepson's third broad contextual dimension is labelled cultural and contains two suggested components of national culture and organizational culture. Within our study, however, we found that participants spoke about particular issues which might be categorised as part of a 'professional culture' and this informed their views of what leadership in nursing might look like or aspire to. The data in this theme are relatively complex and overlap considerably with other themes. However, we perceive that the combination of clinical expertise with a tradition in which nurses provide both instrumental and communicative care to patients provides a distinctive context with a 'professional values' overlay that informs views on the approaches to leadership.

Extract 36: What should nursing leaders aspire to? 
Several participants spoke about the type of leadership they experienced and the practices that they felt should be aspired to by nurses. Some comments were relatively general and, while they provide insights into preferred leadership practices, might be considered as applicable to a range of contexts.

Extract 37: Empowerment of people, making them feel their work is worthwhile...a voice in the organization.

Extract 38: Taking people along with you...knowing.... what is the doing stuff to make that person work more effectively for you.

Other comments, however, were more closely related to the perceived need for nursing leaders to combine leadership practices with clinical expertise. The tension between nurse leaders and clinical nurses has been described by Bolton (2004) as the 'we/they' dichotomy. Clinical nurses may believe leaders are out of touch with clinical practice and make decisions based on financial or political expediency rather than on concern for patients or staff. This would suggest clinical practice is an indicator of membership of the 'in-group' and thus provides the legitimacy to exercise the power of leadership (Hogg, 2001; Cicero et al. 2010). On this point there appeared to be 
tension between the expectation that nurses with clinical expertise might be expected to practice, and the organizational hierarchies in which they work.

Extract 39: Very command and control kind of organization... respect is shown for those leaders because the nursing structure is so embedded.

Extract 40: Hierarchical model... CNS does a lot of good work but they still look for permission to do things.

Extract 41: they come in as a base level nurse and they see what the structure is, and the leadership is very defined for them.

Extract 42: [Leadership requires]...integrity, open door, be approachable, clinical expertise.

Some participants involved specifically in midwifery roles made the case that there are special leadership issues for midwives related to their culture of autonomous practice. One issue noted is that 'nursing 'leads' the process around the development of clinical plans' which is a very different relationship to that of the midwife. 
Extract 43: Very much [an issue] because a midwife is really educated to be an autonomous practitioner...nursing is generally providing care within the medical model...midwifery is actually about a wellness model.

To some extent there is an overlap between this theme and the data in the theme that examined participants' views on the tension between leadership and management in their organization. The 'cultural' dimension of nursing, with a tradition of clinical expertise and autonomy appears to be an important contextual feature that needs to be closely integrated with our understanding of leadership in this sector.

\section{Discussion - revisiting nursing and leadership literature through a contextual lens}

The findings from our project, together with a systematic focus on contextual dimensions led us to revisit previous research on nursing with a view to identifying literature that may provide additional insights into the perceptions and experiences related by participants in our study. Our review of the literature led us to identify two key areas of existing research in nursing which provide potential insights into the emergent themes from our research. The relative neglect of contextual dimensions in previous research has meant that these issues are not clearly integrated into understanding of leadership practice among nurses in Western Australia. The 
identified areas of research were: (i) dissonance between professionalism and managerialism and (ii) implications of hierarchical organizational structures. Viewed within an explicit focus on the importance of context and the interaction of numbers of contextual factors for leadership practice, the links between these and nursing leadership become clearer.

\section{Professionalism and managerialism}

We turn firstly to the apparent dissonance between professionalism and managerialism. In their 2002 study of Western Australian nurses, Nowak and Bickley (2005: 422) identified 'an ideological dissonance with their environment', 'a clash between the commercial and clinical cultures in the WA health system'. The authors analysed the results of their study using the framework of New Public Sector Management (NPM) and managerialism, and argued that the requisites of the public health sector administrative reform implemented in the 1980s are not conducive with the professionalism that underpins the delivery of the healthcare system. Their findings resonate with similar studies in other 'caring professions' such as social work (e.g .Harris, 2003; Lymbery, 2000; May and Buck, 2000). The dissonance identified also relates to what Tummers, Bekkers and Steijn (2009) define as 'policy alienation', or psychological disconnect from the policies being implemented. While there is some 
suggestion in the literature that the discussion around NPM is now muted and we are now in the 'post-NPM' era (Doessel, Williams and Whiteford, 2009), we believe that the effects of NPM are still to be felt within the sector and this is borne out by our research.

Underpinning the managerialist philosophy 'is the belief that previous forms of 'bureau-professional' organization did not provide an effective form of management, and that the presumed technical rationality of managerialism is to be preferred to the indeterminate processes which were held to characterise professionally dominated decision making' (Lymbery, 2000: 128). The introduction of managerialism 'was accompanied by a new vocabulary of 'performance indicators', 'deliverables', 'targets', 'devolved budgets', 'organizational development teams', 'objectives' and 'evaluation schemes" (May and Buck, 2000: 142). Its aim was to make sector organizations and their employees more business-like and market-oriented, 'that is, performance-, cost-, efficiency- and audit-oriented' (Diefenbach, 2009: 893). 'Value for money', 'what works', and positions in league tables provide primary frames of reference'. What we see in the nursing literature, which is consistent with our data, is continuing confusion, discomfort with nursing leadership or a perceived lack of leadership, and role ambiguity. Nowak and Bickley (2005: 417) identified conflict 'between the personal and professional value systems of nurses and the culture of their healthcare 
organizations operating in a climate of managerialism'. 'Truly compassionate care is skilled, competent, value-based care' (Prime Minister's Commission, 2010: 3) and there are 'core values of wanting to make a difference to patient care' (Queensland Health, 2008: 4).

The International Council of Nurses (Royal Australian College of Nursing, 2011) is guided by five core values - visionary leadership, inclusiveness, flexibility, partnership and achievement, while the core values espoused by the American Association of Colleges of Nursing are human dignity, integrity, altruism, autonomy, and social justice (Shaw and Degazon, 2008). These are the values that are in conflict with the requirements of managerialism and healthcare systems which may not commit to them. Sellman (2011: 203) asserts that the 'marginalisation of nursing's voice is compounded by the rhetorical value claims among many managers and decision-makers in healthcare at all levels'. In making decisions that discourage rather than encourage practitioner values, they are either indulging in self-deception or deliberately maintaining 'a fiction of upholding practitioner values while knowingly undermining the possibilities for those values to thrive' (Sellman, 2011: 204). Professional practice is more than the mastery and performance of a set of tasks, there must be a certain 'something else' to it, and it is the difficulty in defining this 'something else' that conflicts with empiricist categorization (Sellman, 2011). 
Consistent with our findings, an evaluation of the role of matrons in National Health Service Trusts in the UK, found a number of matrons were worried about their lack of direct involvement in patient care and apologised for not being 'proper' matrons. Their 'failure to match up to idealised expectations of the matron role was clearly a source of anxiety' for some participants (Royal College of Nursing Institute 2004: 113). Sixty-percent of the participants reported a lack of clarity about their role - either they were unclear about the role themselves or there were misunderstandings about their role by other staff, which they felt undermined their effectiveness. Ward sisters interviewed felt that the priority given by matrons to organizational/managerial concerns 'diluted their clinical role' and that matrons were too far removed from patient care (Royal College of Nursing Institute, 2004: 115). The contrast between the needs of managerialism and professionalism means that nurses do not see managers as leaders because they are away from the professional decision making role and lack the autonomy of clinical practitioners.

Paliadelis (2005) found an unwillingness amongst Nurse Unit Managers to identify with the role of 'manager', instead perceiving their role as primarily that of a nurse. 'The NUMs tended to devalue and discount the administrative and managerial aspects of their job, preferring to talk about their nursing role' (Paliadelis, 2005: 5). Paliadelis's study took place across small towns in New South Wales, in hospitals 
where nursing staff had worked together for many years and where NUMs had usually been promoted from the wards. Her description of the sense of isolation felt by NUMs and the difficulty they experienced maintaining a professional distance from their colleagues may explain their reluctance to identify with the 'other' or managerial/leadership role.

The Prime Minister's Commission on the Future of Nursing and Midwifery in England (2010: 86) also identified the lack of recent clinical experience of some nurse leaders as an issue. Some respondents to the Commission said that this 'could result in a lack of clinical credibility and awareness and hinder them from taking a lead role in clinical governance'.

The tension between nurse leaders and clinical nurses has been described by Bolton (2004) as the 'we/they' dichotomy. Clinical nurses may believe leaders are out of touch with clinical practice and make decisions based on financial or political expediency rather than on concern for patients or staff. Leadership knowledge and skills are devalued and while leaders may be tolerated, they are also seen as barriers to nurses fulfilling their own needs. We find the descriptive experiences of nursing leadership can be partly explained in terms of Hogg's Social Identity Theory of 
Leadership where the leader's legitimacy is bound up with being prototypical of the group (Hogg, 2001).

Diefenbach (2009: 903) suggests that professionals in management, "have set aside their professional worldviews and values, and have adapted themselves to managerial attitudes, rhetoric and ideology'. One effect of this co-option can be that professional leaders become an 'out group', differentiated from the 'in-group' by attitudes and behaviours that are not seen as in tune with the values of the profession. This perspective is supported by the social identity theory (Tajfel, 1978) of leadership. Hogg (2001: 186) suggests leadership dynamics 'may be significantly affected by the social cognitive processes associated with group membership' such as selfcategorization (assimilating attitudes, feelings and behaviours consistent to the 'ingroup') and depersonalization (context specific prototypes define and prescribe attitudes, feelings and behaviours that distinguish from other groups). 'Leaders and followers are interdependent roles embedded within a social system bounded by common group or category membership' (Hogg, 2001: 186).

This negative view of leadership roles 'is a deterrent to recruiting capable nurses into leadership positions, interferes with effective succession planning, isolates leaders from those they are trying to lead, perpetuates a culture of blame and conflict, 
spurs leader burnout, creates unsafe environments for staff and patients, and prevents individuals and organizations from achieving their full potential' (Bolton, 2004: 34).

A further result of working within a managerialist health system is the change from a profession that was previously self-managed, to one which includes generic managers who may not understand the needs and issues for nurses (Nowak and Bickley, 2005). However, those balancing the dual role of specialist and manager may also experience conflict because while they may be trained in their profession, they may not be trained in management. In their frustration in dealing with their management responsibilities they may see their 'real work' as their technical specialty and neglect their managerial tasks (McConnell, 2002).

\section{Leadership within hierarchical systems}

Our data suggest that an understanding of the practice and issues for nursing leadership also requires consideration of the historical hierarchical model of nursing leadership and the medical model along with newer contextual developments such as changing practice for working in teams, changing educational models and intergenerational stresses. Nurses work in multidisciplinary teams and nursing leaders work within the hierarchical structures of the hospital system. Nursing leadership is impacted on by the other health professions, particularly medicine. The medical 
model which dominates hospitals causes conflict as today's highly-educated nurses expect a greater professional role than medical doctors may be willing to concede. Traditionally doctors were expected to be self-reliant and independent, with expertise and autonomy emphasised more than interdependence and dialogue. Conversely, nursing traditions have emphasised hierarchical rule following, with nurses working 'around' others (Davies, 2000) to avoid conflict and stress. The resulting avoidance behaviour creates tension for nurses and Churchman and Doherty (2010: 46) suggest that nurses themselves, particularly some senior nurses 'are complicit in their own subordination through their behaviour in supporting deference to the medical profession'. Radcliffe (2000: 1085) goes so far as to suggest that in developing postgraduate education, evidence based practice and expanded roles, nurses are even 'mimicking the medical career structure' themselves, 'in order to redesign their sense of self'. The hierarchical structure of healthcare systems 'accentuates the conviction that power should be concentrated above and that power ultimately benefits from singularity and isolation' (DiPalma, 2004: 305). Medical homophily continues to exist 'despite decades of change aimed at reducing medicine's power' (Lewis, 2006: 2134). The hierarchical 'doctor-nurse game' fosters arrogance, condescension, and inequality and limits the potential of nursing staff (DiPalma, 2004). 'Nurses have to prove their competence in every interaction with physicians, whereas doctors' competencies are 
assumed and it is their fallibility and shortcomings that have to be proved' (Fagin and Garelick, 2004: 282). The gendered division of labour within healthcare may also exacerbate the subordinate position of nurses (Churchman and Doherty, 2010). A study by Atsalos (2004, quoted in Surakka, 2008: 527) found that the main problem for nurse managers in Australia is the hierarchical relationships between the different health professionals. In the UK, the Department of Health (2009: 33) suggests that midwifery leaders can feel isolated in a management structure and that there is a need to move from hierarchical models to building a leadership team. What are the implications of these hierarchical relationships to nursing leadership? Cott (1997: 1418) suggests that as groups which are subordinate to medicine, such as nursing, have sought and achieved greater autonomy and control over the conditions of their work, teamwork has evolved as a more egalitarian, independent model of working. However, her research has found that the interaction that does occur between nursing and the core multidisciplinary professionals, occurs mainly through the higher status nurses'. There are clear differentiations of power and influence between higher status nurses and nursing 'sub-teams', whose teamwork consists of assisting each other with work tasks. Thus while the development of teamwork may have increased the participation of nurses in decision-making, rather than flattening the hierarchical structure of health staffing, its effects may have been limited to high level nurses. 
In a study of senior nurse executives and medical leaders in acute-care hospitals in the UK, West and Barron (2005) found that professional homophily was more marked among doctors than nurses, that 'the boundary around the medical profession is very strong' and 'the consultant network could comprise a powerful block within the hospital' (West and Barron, 2005: 139). They comment on the fact that peer groups appear to be available for Clinical Directors of Medicine but not for Directors of Nursing with hospitals. In terms of leadership, it appears that senior nurse leaders are disadvantaged in the level of peer support available to them.

In an Australian study of the gatekeeping practices of nurses in operating theatres, Riley and Manias (2009: 219) found that 'gatekeeping was used by nurses to negotiate their social positioning in the healthcare hierarchy, both in relation to one another and with their medical colleagues. Nurse managers exercised control of information to reinforce their position in the nursing hierarchy'. The exercise of power and control by the nurses was 'a means through which traditional medical-nursing boundaries change and evolve, and the relationship between the two professions is altered'.

Within professionalism itself there is also discord which is linked to the hierarchical system of nursing and variation in leadership style. In this sense 
'hierarchical' may relate to age differences in addition to formal reporting structures. Duchscher and Cowin (2004) suggest that the micromanagement style of older nursing leaders clashes with the needs of younger nurses who want to work in a culture of independence and collaboration. While responding to positive mentoring, they will resist what they perceive as prescriptive or autocratic leadership styles. Hunter's (2005) study of student midwives and qualified midwives also identified the opposing perspectives of junior and senior staff. The work of senior midwives involved more managerial and administrative responsibility and less hands-on patient care, they 'were responsible for delivering a universal service, and had to consider the 'bigger picture' in a way that junior staff did not'. From the perspective of junior midwives, this led to a 'clear the board' mentality, but senior midwives rationalised their attempt to hasten women's progress through the hospital system as being necessary to meet the hospital's goals of efficiency and task completion (Hunter, 2005: 257). Wilson et al. (2008) suggest that younger nurses be given more opportunities to participate in decision-making regarding nursing care as a way of increasing perceived autonomy and control. There continues to be demand from a new generation of nurses to identify with their profession and to expect to be able to work in an autonomous way. This demand is reinforced by the move from hospital trained nurses to university training and the introduction of higher level professional positions such as nurse practitioner. 
Chadwick (2010: 166) suggests that today's generation of nurse leaders 'faces the challenge of bridging the generation gap between older nurses and physicians, who may have a patriarchal or hierarchical view of healthcare, and the younger generation, which expects everyone to be treated as equals'. The hierarchical view of healthcare and hospital culture was also identified in Hunter's (2005) study. Hospitalbased midwifery was strongly hierarchical and while junior midwives had little overt power, 'the status positions of senior midwives were maintained through unwritten rules, enforced through an informal system of reward and punishment' (Hunter, 2005: 257).

The hierarchical medical model not only dominates the healthcare working environment but also extends to clinical knowledge. While Fagin and Garelick (2004) suggest that nurses are increasingly questioning the narrow approaches of the medical model and focussing on a broader, more holistic approach to healthcare, Coombs and Ersser (2004) suggest that aspects of clinical knowledge tend to be placed within a hierarchy by medical staff. Because medical knowledge remains dominant, clinical decision-making is controlled by doctors. Doctors perceive some aspects of nursing knowledge as 'clinically superficial' and on the periphery of patient management, while at the same time recognising that nurses are essential for the delivery of high quality healthcare. This paradoxical situation means that nurses are 'required to 
manage the tensions of adopting the dual institutional roles of self-disciplined assistants and professionally educated practitioners' (Coombs and Ersser, 2004: 250).

The specific contextual issues identified in our research and detailed in the diagram below, are consistent with the Jepson identification of three broad interacting contextual influences on leadership.

Insert Figure 2 here

\section{Conclusion}

Our data collection and analysis, coupled with a systematic investigation of contextual dimensions of nursing in Western Australia suggests that there is considerable scope for developing context contingent models of leadership. Such an approach is very different from traditional models of leadership that typically draw from individualised accounts of agency. It represents a contrast by focusing on the interplay between organizational or institutional context and the manner in which individual actions can be constrained or change the contexts in which they take place. In this view of 
leadership, the interaction of contextual dimensions can act as a facilitator or constraint on leadership practice.

Our research of nursing leadership in Western Australia suggests that previous, 'universal' theories of leadership practice may provide some insights into leadership practice. However, without a similar focus on the interaction of the social, institutional and cultural contexts in which leadership takes place, our understanding of nursing leadership will remain limited and it remains difficult to understand key issues such as the lack of status accorded to important management roles relative to clinical roles among nurses.

In a similar vein, our use of Jepson's contextual framework in our analysis suggests the importance of being alert to contextual dimensions that might not be present in expository diagrams such as that included at the start of this paper. While providing a helpful framework, key contextual factors such as geographic features of Western Australia and intergenerational concerns in nursing education and socialization may not be identified. This again, suggests that overarching models, while helpful, require flexibility and adaptability if they are to provide useful analytical tools across contrasting context of leadership practice.

\section{Acknowledgements}


The authors gratefully acknowledge the valuable contribution made to this project by the Office of the Chief Nurse and Midwife and study participants representing various stakeholders in Western Australia's public health sector. Therese Jefferson gratefully acknowledges the support of Edith Cowan University where she was hosted as visiting scholar while co-authoring revisions to this article.

\section{Funding acknowledgement}

This work was supported with funding provided by the Office of the Chief Nurse and Midwife.

\section{References}

Bolden R (2011) Distributed leadership in organizations: a review of theory and research. International Journal of Management Reviews 13(3): 251-269.

Bolton JG (2004) Valuing leadership: transforming the "We/They" dichotomy in nursing organizations. Nurse Leader 2(4): 33-35.

Calpin-Davies PJ (2003). Management and leadership: a dual role in nursing education. Nurse Education Today 23(1): 3-10.

Canadian Nurses Association (2009) Position Statement: nursing leadership. Available at: 
http://www.cnanurses.ca/CNA/documents/pdf/publications/PS109_Leadership 2009

e.pdf.

Carney M (2009) Public Health Sector nurses perception of clinical leadership in Ireland: narrative descriptions. Journal of Nursing Management 17(4): 435-445.

Carryer J, Gardner G, Dunn, S and Gardner, A (2007) The core role of the nurse practitioner: practice, professionalism and clinical leadership. Journal of Clinical Nursing 16(10): 1818-1825.

Chadwick MM (2010) Creating order out of chaos: a leadership model. AORN Journal 91(1): 154-170.

Churchman JJ and Doherty C (2010) Nurses' views on challenging doctors' practice in an acute hospital. Nursing Standard 24 (40): 42-47.

Cicero L, Pierro A and van Knippenberg D (2010) Leadership and uncertainty: how role ambiguity affects the relationship between leader group prototypicality and leadership effectiveness. British Journal of Management 21: 411-421.

Coombs M and Ersser SJ (2004) Medical hegemony in decision-making - a barrier to interdisciplinary working in intensive care? Journal of Advanced Nursing, 46 (3): 245252.

Cott C (1997) "We decide, you carry it out": a social network analysis of multidisciplinary long-term care teams. Social Science \& Medicine 45(9): 1411-1421. 
Cummings G, Lee H, MacGregor T, Davey M, Wong C, Paul L and Stafford, E (2008) Factors contributing to nursing leadership: a systematic review. Journal of Health Services Research \& Policy 13(4): 240-248.

Davidson PM, Elliott D and Daly J (2006) Clinical leadership in contemporary clinical practice: implications for nursing in Australia. Journal of Nursing Management 14(3): 180-187.

Davies C (2000) Getting health professionals to work together. British Medical Journal 320(7241):1021.

Department of Health (2009) Delivering high quality midwifery care: the priorities, opportunities and challenges for midwives. London: Department of Health.

Diefenbach T (2009) New Public Management in public sector organizations: the dark sides of managerialistic 'enlightenment'. Public Administration 87(4): 892-909.

DiPalma C (2004) Power at work: navigating hierarchies, teamwork and webs. Journal of Medical Humanities 25(4):291-308.

Doessel DP, Williams RFG and Whiteford H (2009) Deinstitutionalisation and managerialism in Queensland's psychiatric institutions: challenging 'rhetoric' with empirical results. The Australian Journal of Administration 68(4): 459-483.

Duchscher J EB and Cowin L (2004) Multigenerational nurses in the workplace. JONA 34(11): 493-501. 
Fagin L and Garelick A (2004) The doctor-nurse relationship. Advances in Psychiatric Treatment 10(4): 277-286.

Grint K (2005) Leadership: Limits and Possibilities. London: Palgrave McMillan.

Harris J (2003) Professionalism takes a pounding: the impact of new managerialism on social work in Britain. In: Muetzelfeldt $\mathrm{M}$ and Briskman $\mathrm{L}$ (eds) Moving beyond managerialism in human services. Melbourne: RMIT Publishing, 5-22.

Hogg MA (2001) A social identity theory of leadership. Personality and Social Psychology Review 5(3): 184-200.

Hunter B (2005) Emotion work and boundary maintenance in hospital-based midwifery. Midwifery 21(3): 253-266.

Jackson B and Parry KW (2008) A very short, fairly interesting and reasonably cheap book about studying leadership. Los Angeles, London: Sage.

Jepson D (2009) Leadership context: the importance of departments. Leadership \& Organization Development Journal 30(1): 36-52.

Ladkin D (2010) Rethinking leadership: a new look at old leadership questions. Cheltenham: Edward Elgar.

Laurent C L (2000) A nursing theory for nursing leadership. Journal of Nursing Management 8(2): 83-87. 
Lewis JM (2006) Being around and knowing the players: networks of influence in health policy. Social Science \& Medicine 62(9): 2125-2136.

Lymbery M (2000) The retreat from professionalism: from social worker to care manager. In: Malin N (ed) Professionalism, boundaries and the workplace. London: Routledge, 123-138.

McArthur DB (2006) The nurse practitioner as leader. Journal of the American Academy of Nurse Practitioners 18(1): 8-10.

McCallin AM and Frankson C (2010) The role of the charge nurse manager: a descriptive exploratory study. Journal of Nursing Management 18(3): 319-325.

McConnell CR (2002) The health care professional as a manager: finding the critical balance in a dual role. Health Care Manager 20(3): 1-10.

May T and Buck M (2000) Social work, professionalism and the rationality of organisational change. In: Malin N (ed) Professionalism, boundaries and the workplace. London: Routledge, 139-157.

New South Wales Health (2009) Take the lead: strengthening the nursing/midwifery unit manager role across NSW. Report phase one: August 2008. North Sydney: Department of Health.

Nowak M and Bickley M (2005) Nurses' voices. Australian Bulletin of Labour 31(4): 407-425. 
Osborn, RN, Hunt, JG and Jauch, LR (2002) Toward a contextual theory of leadership. Leadership Quarterly 13: 797-837.

Paliadelis PS (2005) Rural nursing unit managers: education and support for the role. The International Electronic Journal of Rural and Remote Health Research, Education, Practice and Policy 5(1): 325.

Pinch C and Della P (2001) The West Australian study of nursing and midwifery: new vision new direction. Perth: Department of Health.

Prime Minister's Commission on the Future of Nursing and Midwifery in England (2010) Front line care: report by the Prime Minister's Commission on the Future of Nursing and Midwifery in England. London: the Commission.

Queensland Health (2008) Review of the nurse unit manager role: final report. Available at: http://www.health.qld.gov.au/ocno/documents/numreport.pdf.

Radcliffe M (2000) Doctors and nurses: new game, same result. British Medical Journal 320 (7241): 1085.

Riley R and Manias E (2009) Gatekeeping practices of nurses in operating rooms. Social Science \& Medicine 69(2): 215-222.

Royal Australian College of Nursing (2011) International Council of Nurses. Available at: http://www.rcna.org.au/Default.aspx?SiteSearchID=360\&ID-/results. 
Royal College of Nursing Institute (2004) Evaluation of the modern matron role in a sample of NHS trusts: final report to the Department of Health. Available at:

http://www.ren.org.uk/ data/assets/pdf file/007/78640/002493.pdf.

Sarros JC (2009) Contemporary perspectives on leadership: focus and meaning for ambiguous times. Prahran: Tilde University Press.

Sellman D (2011) Professional values and nursing. Medicine, Healthcare and Philosophy 14(2):203-208.

Shaw HK and Degazon C (2008) Integrating the core professional values of nursing: a profession, not just a career. Journal of Cultural Diversity 15(1):44-50.

Stanley D (2006a) In command of care: clinical nurse leadership explored. Journal of Research in Nursing 11(1): 20-39.

Stanley D (2006b) Role conflict: leaders and managers. Nursing Management 13(5): 3137.

Surakka T (2008) The nurse manager's work in the hospital environment during the 1990s and 2000s: responsibility, accountability and expertise in nursing leadership. Journal of Nursing Management 16(5): 525-534.

Tajfel H (Ed.) (1978) Differentiation between social groups: studies in the social psychology of intergroup relations. London: Academic Press. 
Tummers L, Bekkers V and Steijn B (2009) Policy alienation of public professionals: application in a New Public Management context. Public Management Review 11(5): 685-706.

Watson C (2008) Assessing leadership in nurse practitioner candidates. Australian Journal of Advanced Nursing 26(1): 67-76.

West E and Barron DN (2005) Social and geographical boundaries around senior nurse and physician leaders: an application of social network analysis. Canadian Journal of Nursing Research 37(3): 132-148.

Williams AK, Parker, VT, Milson-Hawke S, Cairney K and Peek C (2009) Preparing clinical nurse leaders in a regional Australian teaching hospital. The Journal of Continuing Education in Nursing 40(12): 571-576.

Wilson B, Squires M, Widger K, Cranley L and Tourangeau A (2008) Job satisfaction among a multigenerational nursing workforce. Journal of Nursing Management. 16(6): 716-723.

Woods NF (2003) Leadership - not for just a few. Policy, Politics, \& Nursing Practice 4(4): 255-256.

Associate Professor Linley Lord is Director, Maureen Bickley Centre for Women in Leadership; Director, Teaching and Learning, Curtin Graduate School of Business. Dr Lord has considerable experience as a change agent through appointments such as Project Officer for Equal Employment Opportunity in Local Government (WA); the first Equal Opportunity Co-ordinator for the West Australian Fire Brigades Board (now 
FESA\}; Equal Opportunity Manager for the Reserve Bank of Australia and Equity and Diversity Manager and Director of the Women in Leadership Project at Edith Cowan University. Linley's academic contributions in recent years include being the Director of the Curtin Graduate School of Business Master in Business Leadership program; Chair of the Faculty's Strategic Review Board and coordinator of numerous Masters levels courses in Organizational Behaviour, Scenarios and Strategies and Futures and Foresight Studies. She also has over 15 years' technical experience gained in research and teaching laboratories in diverse areas including soil research, cancer research, physiology and anatomy teaching.

Associate Professor Therese Jefferson is a Senior Research Fellow with the Curtin Graduate School of Business and the Women in Social \& Economic Research (WiSER) unit at Curtin University. She has been an active participant in WiSER research projects since the unit's formation in 1999. She has a record and continuing interest in gendered analysis of labour market issues and plural research methods in economic research. Therese's research has been published in a range of national and international journals and other forums, including the Cambridge Journal of Economics, Australian Bulletin of Labour, Feminist Economics, Journal of Economic Methodology, Economic Record, Journal of Australian Political Economy, Labour and Industry, Journal of Industrial Relations and the Encyclopedia of Political Economy.

Associate Professor Des Klass has a vision to make a positive and lasting difference to the organizations with whom he is involved and to see Australian organizations adding value to their activities by leveraging the extremely useful decision support technologies that are now accessible. To this end he has established the Strategic Planning and Decisions Unit and the Stratcom Facility which focuses on developing specialised processes and software for supporting business needs in strategic planning activities, resource allocation and decision making. Des has extensive experience in consultancy and contract research projects. He is responsible for teaching research 
methods to the Curtin Business School's doctoral students and has extensive teaching experience with Curtin Graduate School of Business's MBA program.

Professor Margaret Nowak is an economist. She was the founding Director and Head of School at the Curtin Graduate School of Business from 1993 to 2003. Since then Margaret has undertaken research and doctoral supervision and is a director of the School's Governance and Corporate Social Responsibility Research Unit. Margaret's long term research interests have focused on labour markets and on corporate governance. In recent years Margaret has undertaken extensive research and consultancy work on the nursing labour market. In 2001-2003 Margaret was the research leader for an Australian Research Council funded project investigating he nursing labour market. In the field of Corporate Governance Margaret has undertaken research on information issues in accountability, shareholder activism and governance, comparative governance and corporate social responsibility. She has been involved in two submissions to The Joint Parliamentary Committee, Corporations and Markets, Commonwealth of Australia. Margaret has held/holds a number of community board positions

Ms Gail Thomas is an experienced manager, researcher, writer and policy analyst within the public, private and academic sectors, including three years running her own research consultancy. Gail has worked in the Commonwealth and State health departments, specialising in primary health, indigenous health and workforce issues. She has also been a Principal Policy Advisor to two State Ministers for Health. Gail's positions in academia include research officer with the WA Centre for Evidence Based Nursing (Curtin School of Nursing) and researcher in the Women in Leadership Project (ECU). As Manager of the Governance and Corporate Responsibility Research Unit at the Curtin Graduate School of Business, Gail undertook projects with Professor Nowak for the Curtin School of Nursing in 2008-09, Employment Directions of Curtin School of Nursing and Midwifery Graduates and The Barriers to Returning to Work after 
Maternity Leave for Health Professionals. Gail is currently researching and publishing on nursing workforce and leadership issues.

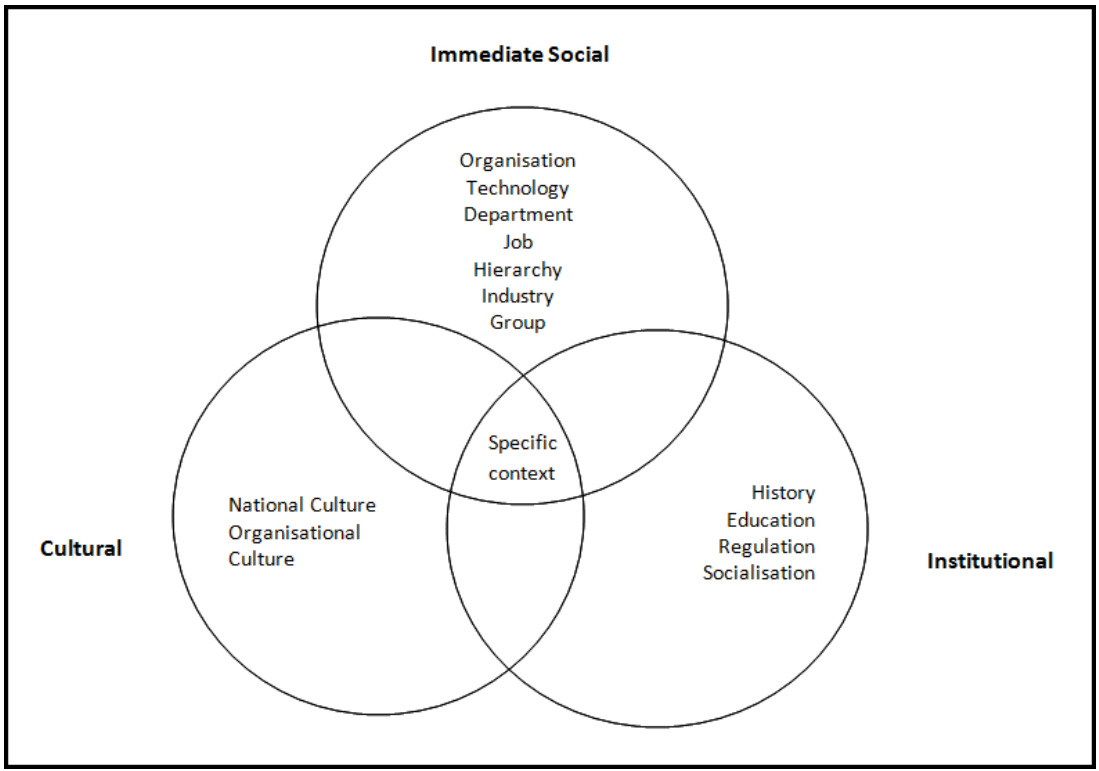

Figure 1: A dynamic interaction of different levels and types of context

Source: Jepson, 2009. 


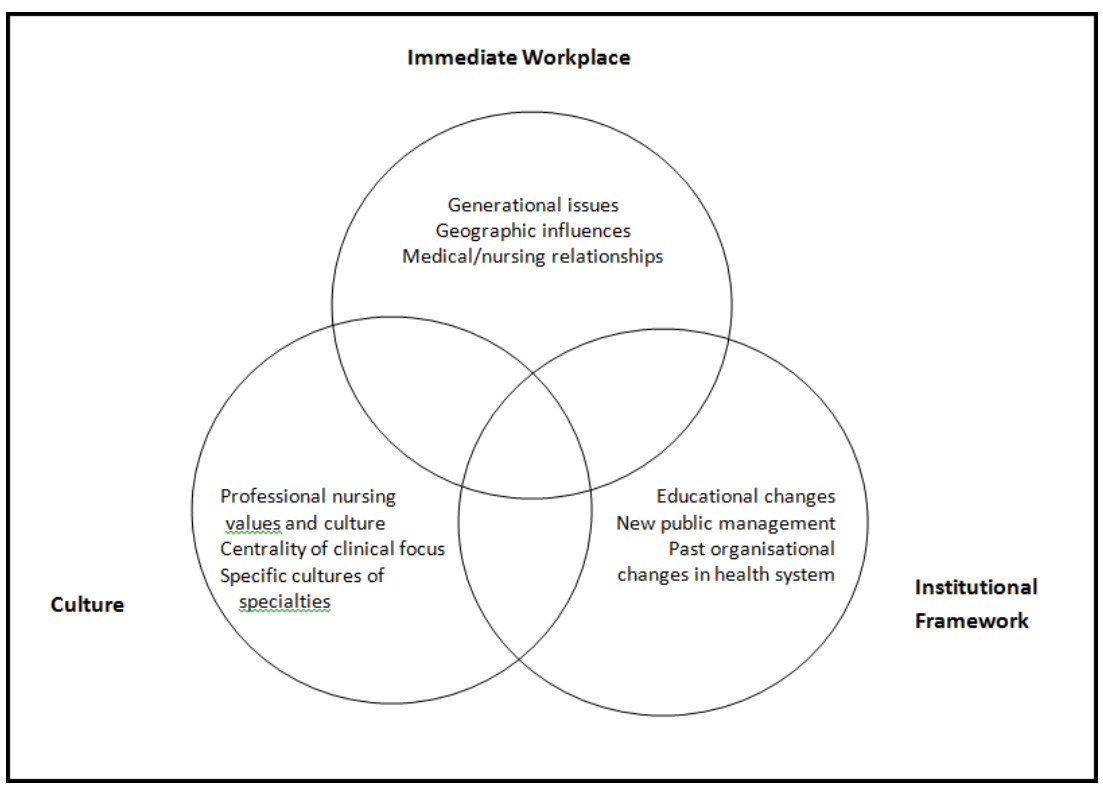

Figure 2: Context and contextual interactions relevant to nursing leadership

Source: Adapted from Jepson 2009. 